AperTO - Archivio Istituzionale Open Access dell'Università di Torino

\title{
Resolvins: Emerging Players in Autoimmune and Inflammatory Diseases
}

\section{This is a pre print version of the following article:}

Original Citation:

Availability:

This version is available http://hdl.handle.net/2318/1729023

since 2020-02-20T11:04:44Z

Published version:

DOI:10.1007/s12016-019-08754-9

Terms of use:

Open Access

Anyone can freely access the full text of works made available as "Open Access". Works made available under a Creative Commons license can be used according to the terms and conditions of said license. Use of all other works requires consent of the right holder (author or publisher) if not exempted from copyright protection by the applicable law. 


\title{
Potential Roles of Resolvins in Chronic Inflammatory and Autoimmune Diseases
}

\begin{abstract}
:
Specialized Proresolving Mediators (SPMs) including resolvins are metabolic products of omega- 3 fatty acids, and they are synthesized during the initial phases of acute inflammatory responses to promote the resolution of inflammation. Resolvins are produced for termination of neutrophil infiltration, stimulation of the clearance of apoptotic cells by macrophages, and promotion of tissue remodeling and homeostasis. Metabolic dysregulation due to either uncontrolled activity of pro-inflammatory responses or to inefficient resolution of inflammation results in chronic inflammation and may also lead to atherosclerosis or other chronic diseases. The pathogenesis of such diseases involves a complex interplay between the immune system, environmental factors (non-infectious or infectious), and critically depends on individual susceptibility to such factors. The diseases include, for example, rheumatoid arthritis, psoriasis, systemic lupus erythematosus, vasculitis, inflammatory bowel diseases, and type 1 diabetes mellitus. In the present review, resolvins and their roles in the resolution of inflammation, as well as the role of these mediators as potential therapeutic agents to counteract specific chronic diseases are discussed.
\end{abstract}

Keywords: autoimmune diseases, resolution of inflammation, resolvins, specialized proresolving mediators 


\section{1: Introduction}

Acute inflammation is a normal protective response when host cells are affected by injury or microbial pathogen invasion (1). In this regard, uncontrolled inflammatory responses may lead to systemic and chronic inflammatory disorders including neurological disorders, cardiovascular diseases, periodontal diseases, asthma, rheumatoid arthritis, diabetes mellitus, as well as the inflammatory bowel disease (IBD) (1-3). Recent studies have identified the resolution of inflammation as an active process, which is regulated by biochemical mediators and specialized pro-resolving mediators (SPMs), which provides a useful novel basis for our understanding of the principles of recognition and treatment of these inflammatory disorders (4).

On the whole, acute inflammatory responses appear with various signs of inflammation such as pain, swelling, redness, heat, and loss of function (5), which are then followed by the release of chemical messengers (cytokines and chemokines) and pro-inflammatory lipid mediators such as prostaglandins (PGs) and leukotrienes (LTs). The release of these molecules leads to an attack against the triggering agents and to initiation of repair of the injured tissues (6-7). This is accompanied by chemotaxis and recruitment of polymorphonuclear neutrophils (PMNs) and by accumulation of monocyte-derived macrophages which trigger and sustain the local or systemic inflammatory tissue reaction (1). The initial pro-inflammatory cellular reactions also stimulate the biosynthesis of specific omega-3 polyunsatuated fatty acidderived SPMs or resolvins which promote the resolution of inflammation (8). In spite of the essentially protective function of acute inflammation, defective termination or prolonged acute inflammation results in chronic inflammation that can be the root cause of many disorders due to the failure of inflammation resolution (6).

The resolution of an acute inflammatory reaction is characterized by termination of PMN penetration into the infected or injured tissue and by proper apoptosis of the already infiltrated PMNs along with effective efferocytotic clearance by macrophages of the apoptotic PMN, of other apoptotic cells, and of any cellular debris $(7,8)$. The inflammatory process involves many mediators, of which some have pro-inflammatory (prostaglandins and leukotrienes) and others both anti-inflammatory and pro-resolving functions (resolvins, lipoxins, protectins, etc) $(4,9)$. All of these mediators could serve as potential targets for therapeutic actions. Along with lipoxins, the resolvins (resolution phase interaction products), a new family of lipid mediators,, have a variety of functions including anti-inflammatory, proresolving, and neuroprotective effects against various disorders (9).

\section{2: Role of lipid mediators in inflammatory responses}

The inflammatory response to injured tissues involves the liberation of chemical signals in the form of chemokines, cytokines, and lipid-derived mediators by the cells of the innate immune system; initially the PMNs, and then macrophages, mast cells, and endothelial cells, ultimately resulting in an inflammatory defence to protect the affected cells (10). Modulation of the initiation phase of acute inflammation is controlled by a category of lipid mediators such as PGs, LTs, and eicosanoids that are constituted from arachidonic acid (AA, omega-6) and have the efficiency to regulate endothelial permeability and PMN infiltration (11). The initial mediators, like leukotriene B4 (LTB4) and PGs, have key roles to lead the pro-inflammatory 
phases with a maximum number of PMNs within seconds and minutes after the injury (10) (Figure $1 \mathrm{a}, \mathrm{b}$ ).

The resolution of inflammation is a secondary defence mechanism that can be controlled by the initial pro-inflammatory agents at the onset of the inflammation phase. The resolution step is controlled by the local release of different SPMs such as lipoxins, resolvins, protectins, and maresins, collectively known as theSPMs (15-16). Thus, the biosynthesis of these mediators depends on the initial pro-inflammatory stimuli that trigger compensatory mechanisms for the resolution of inflammation (10).

Resolvins and protectins can be generated along different pathways by various immune cells, such as macrophages and PMNs, from the following polyunsaturated fatty acids (PUFAs): eicosapentaenoic (EPA) and docosahexanoic (DHA) acids $(10,12)$. Changing the phase to resolution of inflammation, some eicosanoids such as PGE2, and PGD2 provoke the biosynthesis of pro-resolving lipid mediators such as lipoxin A4 (LXA4), deriving from arachidonic acid, that acts as an endogenous "stop" signal to control the resolution phase within hours up to days $(13,14)$. At this stage, the produced SPMs can halt PMN infiltration, reduce the generation of pro-inflammatory mediators, enhance phagocytosis of apoptotic PMNs ("efferocytosis") by macrophages and promote bacterial clearance $(14,15)$. Actually, phagocytosis of dead cells by macrophages results in the biosynthesis of SPMs, which are involved in the processing of apoptotic cell clearance, similar to "find me" and "eat me" signals including ATP, adenosine, and CX3CL during apoptotic cell engulfment $(16,17)$.

SPMs, by binding to $G$ protein-coupled receptors (GPCRs), induce specialized biological actions (Figure 2). It has been clarified that GPCRs, such as chemR23/ERV, are activated by RVE1 and RVE2, while ALX/FPR2 and GPR32/DRV are stimulated by LXA4 and RVD1 (18). Furthermore, GPR32 is activated by RVD5 and RVD3 (19). On the other hand, RVE1 and RVE2 are considered as kinds of endogenous receptor antagonists for the LTB4 receptor BLT1, which has the capacity to organize PMN trafficking and NF-KB activation at the site of inflammation. RVE1 is considered as an agonist of chemR23 on monocytes and dendritic cells, and thereby stereo-selectively transduces signals to these cells (18), along with the capability to reduce the expression of IL-6 and IFN- $\gamma$ and to inhibit the migration of dendritic cells and cytokine secretion (14). Besides the capacity to inhibit PMN recruitment and promotion of macrophage phagocytosis, SPMs are also able to enhance the host defence through the efflux of phagocytes from inflamed tissues to the lymphatics (20). Either a hyperactive proinflammatory responses or an inefficiency to stimulate resolution, which results from the disordered function of mediators, are prototypical of chronic inflammatory disorders such as atherosclerosis, diabetes, and arthritis $(21,22)$ REF.

\section{3: Resolvins and their targets}

Serhan and his co-workers pioneered the concept that the local response to acute inflammation activates specific biochemical and cellular programs of resolution (7, 23-25). These mediators include the D-series resolvins (RVD1, RVD2, RVD3, RVD4, and RVD5) derived from DHA $(26,27)$, and the E-series resolvins (RVE1, RVE2 and RVE3) that are derived from EPA $(28,29)$ (Figure 3$)$. The detection of resolvins along with other lipid mediators such as 
maresins and protectins has been particularly important since these lipid mediators have been demonstrated to support human health (30) by mitigating inflammation (31) in various pathological conditions, notably in diseases with predominantly autoimmune etiopathogenesis $(13,36,38)$, and in cardiovascular diseases (32),, type 2 diabetes mellitus (39) and cancer (40).

Two receptors from the G-protein family (chemR23 and BLT1) are capable of binding to RVE1. The chemR23, which has a high binding affinity for RVE1, is expressed on the surface of macrophages and dendritic cells and is able to stimulate phagocytosis of apoptotic PMNs by macrophages. Herrera et al. found that RVE1 could reduce the penetration and migration of PMNs and inhibit their rolling (34). Furthermore, RVE1 is able to increase the CCR5 expression and reduce the NF-KB signalling (34). Herova et al. indicated that the expression of chemR23 is limited to the surface of naïve macrophages and classical M1 macrophages, while these receptors are not expressed on the surface of M2 macrophages (35). In addition to that, expression of chemR23 RVE1 on the surface of macrophages increases their ability to phagocytose as well as to secrete the anti-inflammatory cytokine IL-10, thereby boosting their capacity to resolve inflammation (35). In a study on a mouse model of pneumonia, the usage of RVE1 reduced pulmonary PMN accumulation, lessened secretion of inflammatory cytokines, improved the clearance of bacterial cells, and enhanced the survival of mice (36). Elkebir et al. (2012) argued that infection was eliminated by RVE1 in mice with acute lung inflammation (37). Via the LTB4 receptor, BLT1, RVE1 is not only able to produce NADPH oxidases and reactive oxygen species but it also increases neutrophil apoptosis leading to their efferocytosis by the macrophages, resulting in the production of phagocytosis-derived metabolic products, such as 19- hydroxy-RVE1, 20- hydroxy-RVE1 and 20- carboxy- RVE with pro-resolution properties in vivo (38). The ability of RVE2s to control PMN penetration and their anti-inflammatory properties was first discovered by $\mathrm{Oh}$ et al. in zymosan-induced rat model of peritonitis, in which they reported that RVE2 was able to inhibit PMN chemotaxis and increase phagocytosis and produce II-10 (48).

The D-series of resolvin family (the RvDs) is another group of lipid-proresolving molecules with the ability to inhibit PMN penetration and to regulate their activities to enhance phagocytosis by macrophages, and to remove and clear apoptotic cells and bacteria in both in vivo and in vitro environments (39-41). A study on the murine model of arterial neointima formation (carotid ligation) showed that the use of RVD2 in these mice recruited macrophages and regulated PMNs. In vitro studies have confirmed a direct impact of SPMs on vascular smooth muscle cells (VSMCs) that includes lower migration in response to platelet-derived growth factor (PDGF) and altered reaction to inflammatory stimuli (e.g. TNFa) (42). According to these results, changes in the interactions between circulating leukocytes and the vessel wall, and direct effects on the phenotype of VSMCs are the probable mechanisms through which SPMs affect the development of neointima in this murine model (42). The treatment by RVD2 and MaR1 on the reduction of infiltrating monocytes/macrophages and PMNs, as well as changes in the macrophage phenotype towards $\mathrm{M} 2$, lead to the resolution of inflammation and to the reduction of neointima after vascular injury $(42,43)$. 
RVDs can also affect target cells through binding to expressed receptors (GPR32-G-protein in coupled receptor, ALX/ FPR2) on the surface of immune cells $(44,45)$. The overexpression of both receptors improves the RVD1macrophage phagocytosis of PMNs, and conversely, the inactivity of these two receptors weakens this action (44). Park et al. approved the inhibitory impact of RVD2 for transient receptor potential (TRP) subtype ankyryn 1 (TRPA1) and vanilloid 1 (TRPV1) that play significant roles in the inflammatory pain responses. (46). Another benefit of using RVD1 has been observed in acute lung injury, in which the lesions are caused by LPS $(47,48)$. In this model RVD1 inhibits the activity of the inflammatory factor NF-KB and thereby inhibits its effect on peroxisome proliferator-activated receptor (PPAR) $\gamma$ and the ensuing inflammatory downstream signalling pathway.

\section{4: Resolvins and Autoimmune Diseases}

Autoimmune diseases are the conditions arising from an abnormal immune response against healthy cells, tissues and organs. Complex mechanisms, involving $T$ cells, the thymus, the bone marrow, lead to the breaking of the immune tolerance (49). In the presence of genetic predisposition and/or environmental triggers, autoreactive $B$ and $T$ cells and autoantibodies could become involved in a pathological inflammatory response, leading to tissue damage (49). Activation of inflammatory mediators contributes to the development of autoimmune diseases such as systemic lupus erythematosus (SLE), rheumatoid arthritis, autoimmune hepatitis and type 1 diabetes mellitus (50). Through the stimulation of a number of signalling pathways, resolvins might favourably impact on this process. They could accomplish by inhibiting leukocyte recruitment to inflammation site, by stimulating non-inflammatory monocyte recruitment, and by activating macrophages to increase the efferocytotic capacity towards apoptotic neutrophils debris from (51). This could lead to resolution of inflammation, repair and regeneration of damaged tissue, and pain relief (63).

Defects in the resolution of inflammation and inflammatory signals increase the risk of autoimmune diseases (52). The onset of the inflammation process causes the production of some pro-inflammatory cytokines such as interleukin-1 (IL-1 $\beta$ ), IL-6, tumor necrosis factor- $\alpha$ (TNF- $\alpha$ ), interferons (IFNs), macrophage migration Inhibitory Factor (MIF) and high mobility group B1 (HMGB1) protein, and, at the same time, in contrast the production of antiinflammatory cytokines such as IL-10, IL-4, and TGF- $\beta(53,54)$. Indeed, an imbalance between pro- and anti-inflammatory cytokines results in tissue damage and inflammatory disorders, and the inability of the immune system to resolve the inflammation results in the continuation of inflammatory processes in autoimmune diseases such as $\operatorname{SLE}(55,56)$. In these diseases, the process of repairing damaged tissues is delayed even after taking anti-inflammatory drugs and immunosuppressive agents. Therefore, the use of resolution-inducing agents such as PUFAs and the metabolites derived from them, such as resolvins, lipoxins, and protectins, has the potential to restore the normal physiological function of damaged tissues and organs (56). Macrophages and monocytes have GPCRs on their surfaces; the subtypes P2ry2, P2ry6, Edg5 have a particularly important role in the regulation of inflammation and cell-mediated immunity (57). Many studies on PUFAs and the the derived-metabolites, including resolvins and lipoxins, indicate that these lipid mediators act as agonists of G-protein that plays an 
important role in the inflammation, leukocyte recruitment, lipid synthesis and glucose homeostasis (58). It can be thus concluded that PUFAs and derivatives thereof, as the antiinflammatory and cytoptotective resolvins, lipoxins, protectins, are capable of inhibiting the pro-inflammatory MIF, IL-2, IL -6, TNF-a, and HMGB1, thus inhibiting inflammation in autoimmune diseases such as rheumatoid arthritis and SLE (56).

Resolvins also have the ability to inhibit cerebral ischemia-reperfusion due to their neuroprotective activity $(59,60)$. Furthermore, since leukotriene B4 (LTB4) is involved in the pathogenesis of inflammatory bowel disease, lipid mediators such as resolvins could be hypothesized as a potential treatment since studies on rats with colitis have shown that RVE1 increases their survival, decreases weight and reduces the severity of histological lesions (61). Studies also indicated that resolvins reduced both the expression of pro-inflammatory genes such as those coding for IL-12, TNF- $\alpha, N F-\mathrm{KB}$, and decreased inducible NO synthesis in models of inflammatory bowel disease (62).

In addition, many studies indicated roles for dendritic cells in the pathogenesis of inflammatory bowel disease because these cells could lead the orientation of T cells towards the inflammation during maturation and increase the inflammation and resistance of $T$ cells through the production of inflammatory cytokines such as IL-12, IL-23 and TNF- $\alpha(63,64)$. Interestingly, it has been found that lipid mediators, such as the resolvins and lipoxins, could play roles in the function of dendritic cells, especially through different receptors, and prevent their orientation towards inflammation. For instance, EPA-derived RVE1 induced the expression of its specific receptor chemR23 on the surface of dendritic cells and inhibited the secretion of IL- 12, thereby impeding inflammation (14).

The secretion of pro-inflammatory molecules. such as IL-1beta and LTB4, is a leading contributor to inflammation and autoimmune diseases such as rheumatoid arthritis and psoriasis $(65,66)$. Trials indicate that the nutritional use of DHA and EPA, abundantly found in fish oil, can reduce the secretion of inflammatory mediators and thus might be useful for treating inflammatory and autoimmune diseases $(31,67)$. Very recent human data support this notion, as high doses of EPA significantly reduced major cardiovascular events in patients at high cardiovascular risk (68).

Human acute inflammatory demyelinating polyradiculoneuropathy (AIDP) disease, the major reason of acute autoimmune neuromuscular paralysis, is provoked by autoimmune invasion to the peripheral nervous system (REF). The experimental autoimmune neuritis (EAN), an animal model of AIDP, has been applied to determine the therapeutic and functional principles of EAN AIDP $(69,70)$. This disease occurs due to the impaired phagocytosis of apoptotic T cells. Normally, the apoptosis of autoreactive T-cells is a process leading to the end of inflammatory responses in EAN, and is necessary following the clearance of apoptotic cells by macrophages to prevent the accumulation of damaged cells e and the spread of infection and inflammation (secondary necrosis) (71). In addition, the clearance of apoptotic cells caused by active macrophages leads to a change in the anti-inflammatory phenotype inducing $T_{h}$-cells towards the $T_{\text {reg }}$ phenotype due to the production of anti-inflammatory cytokines (72-74). Studies have indicated that $\mathrm{T}_{\text {reg }}$ cells and anti-inflammatory macrophages play important roles in the resolution of inflammation. Therefore, there is a need to find ways 
to enhance the phagocytosis of apoptotic cells in the inflamed tissue to resolve the neural inflammation in the acute inflammatory demyelinating polyradiculoneuropathies $d(75,76)$. Through the presence of two receptors, the ALX/FPR2 and GPR32, on the surface of neutrophils and macrophages, RVD1 is able to transmit a signal to a target cell, which is able to regulate the migration of neutrophils, increase phagocytosis of apoptotic T-cells by macrophages (in an ALX/FPR2-dependent manner) and thus reduce the accumulation of apoptotic T-cells (26). Thus, suchRVD1-induced changes increase the expression of TGF- $\beta$ via anti-inflammatory phenotype of macrophages, reduce the pro-inflammatory factors such as TNF- $\alpha$, and enhance the number of $T_{\text {reg }}$ cells, consequently leading to the resolution of inflammation in autoimmune diseases such as the EAN and AIDP $(26,77)$.

\section{5: Concluding Remarks}

The activation of inflammation by a dysregulated immune system is a central mechanism in the development of autoimmune diseases. In the absence of an efficient inhibition of inflammation, the adaptive immune system of the host elicits a strong proinflammatory response. Therefore, inflammation should be controlled at its early stages. Such early control can prevent the occurrence of chronic inflammation, can lead to repair and regeneration of the damaged tissue parts and might thereby delay or counteract the clinical onset of autoimmune diseases. Indeed, as an inherent component of inflammation, the proinflammatory processes are counteracted by resolution-promoting lipid mediators, such as the resolvins derived from PUFAs. These agents can prevent the spread of inflammation and its chronicity in various ways, notably by acting on different receptors expressed on the surface of macrophages, PMNs and dendritic cells.

The therapeutic use of the PUFA-derived lipid mediators during the inflammation process to resolve inflammation and prevent the development of chronic inflammation could be a useful therapeutic option. On the other hand, since incomplete efferocytosis is one cause of autoimmune diseases, by facilitating efferocytosis and activating macrophage cells to efficiently clear of apoptotic cells and cell debris, the lipid mediators can prevent the progression of inflammation and subsequent inflammatory diseases, in particular autoimmune diseases (78).

The connection between lipid mediators and the clearance of leukocytes via apoptosis has been detected by identifying that the release of lysophosphatidylcholine from apoptotic cells could specifically assemble phagocytes to the resolution site (79). The presence of RVE1 receptors on dendritic cells and neutrophils, as well as the essential role of these cells in inflammatory responses could verify the link between the biosynthesis of lipid mediators and their engagement in cellular trafficking and regulation of inflammatory disorders such as inflammatory bowel disease $(14,61)$.

Owing to their ability to stereoselectively regulate and reduce inflammation, the SPMs and their intricate and highly specific molecular pathways are attracting an ever-increasing attention among many researchers. The PUFA-derived lipid mediators as pro-resolving agents are effective and sustained factors that stimulate leukocyte recruitment, PMN apoptosis at the damaged site, and clearance of damaged tissue parts during the inflammation. Therefore, 
these agents are excellent candidates for the treatment of diseases associated with the defective inflammation resolution, and, also for the facilitation of recovery from primary and secondary infections.

Taken together, the SPMs play an important role in the prevention of chronic inflammation, which is the root cause of various chronic diseases. Therefore, an accurate understanding of the molecular mechanisms by which these pro-resolving compounds may prevent or even cure such pathological conditions, should help designing novel strategies to prevent and treat inflammatory and autoimmune diseases without compromising the ability of the host cells to be in charge of the natural host defence. 


\section{Figure legends:}

\section{Figure 1:}

1a: Ideal outcome of inflammation

Tissue injury and trauma trigger an acute inflammatory cascade as a host defensive mechanism. Formation of edema is the first sign produced by infiltration of neutrophils. Then PMN infiltration provokes monocytes and macrophages to remove apoptotic PMNs. s. At the stage of resolution phase, adaptive immune responses ( $T$ and $B$ cells) are initiated simultaneously with tissue regeneration.

1b: Pro-resolving mediators in inflammatory responses

Trauma, tissue injury, and infections initiate the inflammatory process which comprises of cells like neutrophils and the release of many mediators, such as PGE2, PGI2, and LTB4 along with many cytokines and chemokines. The release of PGs leads to the migration of neutrophils from capillary venules. Concurrent with the accumulation of neutrophils, switching of the lipid mediator class takes place, so that the appearance of lipoxin results in the recruitment of non-phlogistic monocytes. Other SPMs, like resolvins, and protectins limit PMN tissue infiltration, stimulate macrophage phagocytosis, and efferocytosis of cellular debris. SPMs and resolvins RV promote the resolution of inflammation and resolve exudates. Also SPMs and resolvins RV suppress chronic inflammation and improve tissue regeneration and wound healing. Dysregulation of any of these mechanisms may cause chronic inflammatory diseases, situation such as diabetes, atherosclerosis, and rheumatoid arthritis.

Abbreviations: prostaglandin (PG), leukotriene (LT), specialized pro-resolving mediator (SPM), polymorphonuclear neutrophil (PMN), (RV)

Figure 2: Proresolving receptors and biological functions of SPMs

RVD1, and LXA4 are agonists for ALX/FPR2. Lipoxin A4, as well as the D-series resolvins, RVD1, RVD3, and RVD5 are agonists for DRV/GPR32. The E-series Resolvins, RVE1 and RVE2, are agonists for ERV/chemR23 and antagonists for the leukotriene B4 receptor, BLT1.

Figure 3: Biosynthetic pathways of resolvin generation

Different kinds of resolvins are derived from n-3 PUFA as EPA and DHA. The biosynthesis of E-series resolvins from EPA is initiated by insertion of molecular oxygen at the $18^{\text {th }}$ carbon site, converting it to bioactive E-series members resolvinE1, resolvinE2, and resolvinE3. D-series resolvin biosynthesis is along with the insertion of molecular oxygen at the $17^{\text {th }}$ carbon site of DHA. The 17-sHPDHA precursor is changed to different intermediates of D-series resolvins including resolvinD1, resolvinD2, resolvinD3, and resolvinD4, each one of which has a specific function. See ref (80).

Abbreviations: PUFA: poly unsaturated fatty acid, DHA: docosahexaenoic acid, EPA: eicosapentaenoic acid.

AS SUGGESTED IN THE COMMENTS: A $3^{\text {RD }}$ FIGURE WOULD BE VERY NICE: IT SHOULD SHOW THE LIGANDS AND THEIR RECEPTORS, PERHAPS ON A CELL SURFACE 


\section{6: References}

1. Kumar V, Abbas AK, Fausto N, Aster JC. Robbins and Cotran pathologic basis of disease, professional edition e-book: elsevier health sciences; 2014.

2. Libby P, Ridker PM, Maseri A. Inflammation and atherosclerosis. Circulation. 2002;105(9):1135-43.

3. Hansson GK, Libby P. The immune response in atherosclerosis: a double-edged sword. Nature reviews immunology. 2006;6(7):508.

4. Serhan CN, Petasis NA. Resolvins and protectins in inflammation resolution. Chemical reviews. 2011;111(10):5922-43.

5. Majno G, Joris I. Cells, tissues, and disease: principles of general pathology: Oxford University Press; 2004.

6. Serhan CN, Brain SD, Buckley CD, Gilroy DW, Haslett C, O'Neill LA, et al. Resolution of inflammation: state of the art, definitions and terms. The FASEB journal. 2007;21(2):325-32.

7. Serhan CN. A search for endogenous mechanisms of anti-inflammation uncovers novel chemical mediators: missing links to resolution. Histochemistry and cell biology. 2004;122(4):305-21.

8. Serhan CN, Ward PA, Gilroy DW. Fundamentals of inflammation: Cambridge University Press; 2010.

9. Schwab JM, Serhan CN. Lipoxins and new lipid mediators in the resolution of inflammation. Current opinion in pharmacology. 2006;6(4):414-20.

10. Crean D, Godson C, editors. Specialised lipid mediators and their targets. Seminars in immunology; 2015: Elsevier.

11. Samuelsson B, Dahlen S-E, Lindgren JA, Rouzer CA, Serhan CN. Leukotrienes and lipoxins: structures, biosynthesis, and biological effects. Science. 1987;237(4819):1171-6.

12. Chiurchiù V, Leuti A, Dalli J, Jacobsson A, Battistini L, Maccarrone M, et al. Proresolving lipid mediators resolvin D1, resolvin D2, and maresin 1 are critical in modulating $\mathrm{T}$ cell responses. Science translational medicine. 2016;8(353):353ra111-353ra111.

13. Levy BD, Clish CB, Schmidt B, Gronert K, Serhan CN. Lipid mediator class switching during acute inflammation: signals in resolution. Nature immunology. 2001;2(7):612.

14. Arita M, Bianchini F, Aliberti J, Sher A, Chiang N, Hong S, et al. Stereochemical assignment, antiinflammatory properties, and receptor for the omega-3 lipid mediator resolvin E1. The Journal of experimental medicine. 2005;201(5):713-22.

15. Chiang N, Fredman G, Bäckhed F, Oh SF, Vickery T, Schmidt BA, et al. Infection regulates pro-resolving mediators that lower antibiotic requirements. Nature. 2012;484(7395):524.

16. Han CZ, Ravichandran KS. Metabolic connections during apoptotic cell engulfment. Cell. 2011;147(7):1442-5.

17. Köröskényi K, Duró E, Pallai A, Sarang Z, Kloor D, Ucker DS, et al. Involvement of adenosine A2A receptors in engulfment-dependent apoptotic cell suppression of inflammation. The Journal of immunology. 2011:1002284.

18. Serhan CN, Chiang N. Resolution phase lipid mediators of inflammation: agonists of resolution. Current opinion in pharmacology. 2013;13(4):632-40.

19. Dalli J, Winkler JW, Colas RA, Arnardottir H, Cheng C-YC, Chiang N, et al. Resolvin D3 and aspirin-triggered resolvin D3 are potent immunoresolvents. Chemistry \& biology. 2013;20(2):188-201.

20. Schwab JM, Chiang N, Arita M, Serhan CN. Resolvin E1 and protectin D1 activate inflammation-resolution programmes. Nature. 2007;447(7146):869.

21. Spite M, Serhan CN. Novel lipid mediators promote resolution of acute inflammation: impact of aspirin and statins. Circulation research. 2010;107(10):1170-84. 
22. Nathan C. Points of control in inflammation. Nature. 2002;420(6917):846.

23. Serhan CN. Resolution phase of inflammation: novel endogenous anti-inflammatory and proresolving lipid mediators and pathways. Annual review of immunology. 2007;25:101-37.

24. Bannenberg G, Serhan CN. Specialized pro-resolving lipid mediators in the inflammatory response: An update. Biochimica et biophysica acta. 2010;1801(12):1260-73.

25. Serhan CN. Novel lipid mediators and resolution mechanisms in acute inflammation: to resolve or not? The American journal of pathology. 2010;177(4):1576-91.

26. Sun YP, Oh SF, Uddin J, Yang R, Gotlinger K, Campbell E, et al. Resolvin D1 and its aspirintriggered 17R epimer. Stereochemical assignments, anti-inflammatory properties, and enzymatic inactivation. The Journal of biological chemistry. 2007;282(13):9323-34.

27. Spite M, Norling LV, Summers L, Yang R, Cooper D, Petasis NA, et al. Resolvin D2 is a potent regulator of leukocytes and controls microbial sepsis. Nature. 2009;461(7268):1287-91.

28. Arita M, Yoshida M, Hong S, Tjonahen E, Glickman JN, Petasis NA, et al. Resolvin E1, an endogenous lipid mediator derived from omega-3 eicosapentaenoic acid, protects against 2,4,6trinitrobenzene sulfonic acid-induced colitis. Proceedings of the National Academy of Sciences of the United States of America. 2005;102(21):7671-6.

29. Serhan CN, Clish CB, Brannon J, Colgan SP, Chiang N, Gronert K. Novel functional sets of lipid-derived mediators with antiinflammatory actions generated from omega-3 fatty acids via cyclooxygenase 2-nonsteroidal antiinflammatory drugs and transcellular processing. The Journal of experimental medicine. 2000;192(8):1197-204.

30. Fetterman JW, Jr., Zdanowicz MM. Therapeutic potential of n-3 polyunsaturated fatty acids in disease. American journal of health-system pharmacy : AJHP : official journal of the American Society of Health-System Pharmacists. 2009;66(13):1169-79.

31. Simopoulos AP. Omega-3 fatty acids in inflammation and autoimmune diseases. Journal of the American College of Nutrition. 2002;21(6):495-505.

32. Harper CR, Jacobson TA. The fats of life: the role of omega-3 fatty acids in the prevention of coronary heart disease. Archives of internal medicine. 2001;161(18):2185-92.

33. Cleland LG, James MJ, Proudman SM. The role of fish oils in the treatment of rheumatoid arthritis. Drugs. 2003;63(9):845-53.

34. Herrera BS, Hasturk H, Kantarci A, Freire MO, Nguyen O, Kansal S, et al. Impact of resolvin E1 on murine neutrophil phagocytosis in type 2 diabetes. Infection and immunity. 2015;83(2):792-801. 35. Herová M, Schmid M, Gemperle C, Hersberger M. ChemR23, the receptor for chemerin and resolvin E1, is expressed and functional on M1 but not on M2 macrophages. The Journal of Immunology. 2015:1402166.

36. Seki H, Fukunaga K, Arita M, Arai H, Nakanishi H, Taguchi R, et al. The anti-inflammatory and proresolving mediator resolvin E1 protects mice from bacterial pneumonia and acute lung injury. The Journal of Immunology. 2010;184(2):836-43.

37. El Kebir D, Gjorstrup P, Filep JG. Resolvin E1 promotes phagocytosis-induced neutrophil apoptosis and accelerates resolution of pulmonary inflammation. Proceedings of the National Academy of Sciences. 2012:201206641.

38. Hong S, Porter TF, Lu Y, Oh SF, Pillai PS, Serhan CN. Resolvin E1 metabolome in local inactivation during inflammation-resolution. The Journal of Immunology. 2008;180(5):3512-9.

39. Serhan CN. Pro-resolving lipid mediators are leads for resolution physiology. Nature. 2014;510(7503):92.

40. Kohli P, Levy BD. Resolvins and protectins: mediating solutions to inflammation. British journal of pharmacology. 2009;158(4):960-71.

41. Mogensen TH. Pathogen recognition and inflammatory signaling in innate immune defenses. Clinical microbiology reviews. 2009;22(2):240-73. 
42. Akagi D, Chen M, Toy R, Chatterjee A, Conte MS. Systemic delivery of proresolving lipid mediators resolvin D2 and maresin 1 attenuates intimal hyperplasia in mice. The FASEB Journal. 2015;29(6):2504-13.

43. Titos E, Rius B, González-Périz A, López-Vicario C, Morán-Salvador E, Martínez-Clemente $\mathrm{M}$, et al. Resolvin D1 and its precursor docosahexaenoic acid promote resolution of adipose tissue inflammation by eliciting macrophage polarization toward an M2-like phenotype. The Journal of Immunology. 2011:1100225.

44. Krishnamoorthy S, Recchiuti A, Chiang N, Yacoubian S, Lee C-H, Yang R, et al. Resolvin D1 binds human phagocytes with evidence for proresolving receptors. Proceedings of the National Academy of Sciences. 2010;107(4):1660-5.

45. Krishnamoorthy S, Recchiuti A, Chiang N, Fredman G, Serhan CN. Resolvin D1 receptor stereoselectivity and regulation of inflammation and proresolving microRNAs. The American journal of pathology. 2012;180(5):2018-27.

46. Park C-K, Xu Z-Z, Liu T, Lü N, Serhan CN, Ji R-R. Resolvin D2 is a potent endogenous inhibitor for transient receptor potential subtype V1/A1, inflammatory pain, and spinal cord synaptic plasticity in mice: distinct roles of resolvin D1, D2, and E1. Journal of Neuroscience. 2011;31(50):18433-8.

47. Wang B, Gong X, Wan J-y, Zhang L, Zhang Z, Li H-z, et al. Resolvin D1 protects mice from LPS-induced acute lung injury. Pulmonary pharmacology \& therapeutics. 2011;24(4):434-41.

48. Liao Z, Dong J, Wu W, Yang T, Wang T, Guo L, et al. Resolvin D1 attenuates inflammation in lipopolysaccharide-induced acute lung injury through a process involving the PPAR $\gamma / \mathrm{NF}-\kappa \mathrm{B}$ pathway. Respiratory research. 2012;13(1):110.

49. Wang L, Wang FS, Gershwin ME. Human autoimmune diseases: a comprehensive update. Journal of internal medicine. 2015;278(4):369-95.

50. Baccala R, Hoebe K, Kono DH, Beutler B, Theofilopoulos AN. TLR-dependent and TLRindependent pathways of type I interferon induction in systemic autoimmunity. Nature medicine. 2007;13(5):543.

51. Mitchell S, Thomas G, Harvey K, Cottell D, Reville K, Berlasconi G, et al. Lipoxins, aspirintriggered epi-lipoxins, lipoxin stable analogues, and the resolution of inflammation: stimulation of macrophage phagocytosis of apoptotic neutrophils in vivo. Journal of the American Society of Nephrology. 2002;13(10):2497-507.

52. Eizirik DL, Colli ML, Ortis F. The role of inflammation in insulitis and $\beta$-cell loss in type 1 diabetes. Nature Reviews Endocrinology. 2009;5(4):219.

53. Serhan CN, Hong S, Gronert K, Colgan SP, Devchand PR, Mirick G, et al. Resolvins: a family of bioactive products of omega-3 fatty acid transformation circuits initiated by aspirin treatment that counter proinflammation signals. Journal of Experimental Medicine. 2002;196(8):1025-37.

54. Chiang N, Arita M, Serhan CN. Anti-inflammatory circuitry: lipoxin, aspirin-triggered lipoxins and their receptor ALX. Prostaglandins, leukotrienes and essential fatty acids. 2005;73(3-4):163-77.

55. Oh DY, Talukdar S, Bae EJ, Imamura T, Morinaga H, Fan W, et al. GPR120 is an omega-3 fatty acid receptor mediating potent anti-inflammatory and insulin-sensitizing effects. Cell. 2010;142(5):687-98.

56. Das UN. Lipoxins as biomarkers of lupus and other inflammatory conditions. Lipids in health and disease. 2011;10:76.

57. Lattin JE, Schroder K, Su AI, Walker JR, Zhang J, Wiltshire T, et al. Expression analysis of G Protein-Coupled Receptors in mouse macrophages. Immunome research. 2008;4:5.

58. Milligan G, Stoddart LA, Brown AJ. G protein-coupled receptors for free fatty acids. Cellular signalling. 2006;18(9):1360-5. 
59. Marcheselli VL, Hong S, Lukiw WJ, Tian XH, Gronert K, Musto A, et al. Novel docosanoids inhibit brain ischemia-reperfusion-mediated leukocyte infiltration and pro-inflammatory gene expression. The Journal of biological chemistry. 2003;278(44):43807-17.

60. Hong S, Gronert K, Devchand PR, Moussignac RL, Serhan CN. Novel docosatrienes and 17Sresolvins generated from docosahexaenoic acid in murine brain, human blood, and glial cells. Autacoids in anti-inflammation. The Journal of biological chemistry. 2003;278(17):14677-87.

61. Arita M, Yoshida M, Hong S, Tjonahen E, Glickman JN, Petasis NA, et al. Resolvin E1, an endogenous lipid mediator derived from omega-3 eicosapentaenoic acid, protects against 2, 4, 6trinitrobenzene sulfonic acid-induced colitis. Proceedings of the National Academy of Sciences. 2005;102(21):7671-6.

62. Weylandt KH, Kang JX, Wiedenmann B, Baumgart DC. Lipoxins and resolvins in inflammatory bowel disease. Inflammatory bowel diseases. 2007;13(6):797-9.

63. Kelsall BL, Leon F. Involvement of intestinal dendritic cells in oral tolerance, immunity to pathogens, and inflammatory bowel disease. Immunological reviews. 2005;206:132-48.

64. Baumgart DC, Metzke D, Schmitz J, Scheffold A, Sturm A, Wiedenmann B, et al. Patients with active inflammatory bowel disease lack immature peripheral blood plasmacytoid and myeloid dendritic cells. Gut. 2005;54(2):228-36.

65. Allen B. Fish oil in combination with other therapies in the treatment of psoriasis. Health Effects of Omega 3 Polyunsaturated Fatty Acids in Seafoods. 66: Karger Publishers; 1991. p. 436-45.

66. Cleland L, James M. Rheumatoid arthritis and the balance of dietary N-6 and N-3 essential fatty acids. British journal of rheumatology. 1997;36(5):513-4.

67. Kremer JM. n-3 fatty acid supplements in rheumatoid arthritis. The American journal of clinical nutrition. 2000;71(1 Suppl):349s-51s.

68. Bhatt DL, Steg PG, Miller M, Brinton EA, Jacobson TA, Ketchum SB, et al. Cardiovascular risk reduction with icosapent ethyl for hypertriglyceridemia. New England Journal of Medicine. 2019;380(1):11-22.

69. Hughes RA, Cornblath DR. Guillain-Barre syndrome. Lancet (London, England). 2005;366(9497):1653-66.

70. Soliven B. Autoimmune neuropathies: insights from animal models. Journal of the Peripheral Nervous System. 2012;17:28-33.

71. Yun JH, Henson PM, Tuder RM. Phagocytic clearance of apoptotic cells: role in lung disease. Expert review of respiratory medicine. 2008;2(6):753-65.

72. Annunziato F, Cosmi L, Liotta F, Maggi E, Romagnani S. The phenotype of human Th17 cells and their precursors, the cytokines that mediate their differentiation and the role of Th17 cells in inflammation. International immunology. 2008;20(11):1361-8.

73. Fadok VA, Bratton DL, Konowal A, Freed PW, Westcott JY, Henson PM. Macrophages that have ingested apoptotic cells in vitro inhibit proinflammatory cytokine production through autocrine/paracrine mechanisms involving TGF-beta, PGE2, and PAF. The Journal of clinical investigation. 1998;101(4):890-8.

74. Kimura A, Naka T, Kishimoto T. IL-6-dependent and -independent pathways in the development of interleukin 17-producing $\mathrm{T}$ helper cells. Proceedings of the National Academy of Sciences. 2007;104(29):12099-104.

75. Luchting B, Rachinger-Adam B, Heyn J, Hinske LC, Kreth S, Azad SC. Anti-inflammatory Tcell shift in neuropathic pain. Journal of neuroinflammation. 2015;12:12.

76. Zhang Z, Zhang ZY, Schluesener HJ. Compound A, a plant origin ligand of glucocorticoid receptors, increases regulatory $\mathrm{T}$ cells and $\mathrm{M} 2$ macrophages to attenuate experimental autoimmune neuritis with reduced side effects. Journal of immunology (Baltimore, Md : 1950). 2009;183(5):308191. 
77. Luo B, Han F, Xu K, Wang J, Liu Z, Shen Z, et al. Resolvin D1 Programs Inflammation Resolution by Increasing TGF-beta Expression Induced by Dying Cell Clearance in Experimental Autoimmune Neuritis. The Journal of neuroscience : the official journal of the Society for Neuroscience. 2016;36(37):9590-603.

78. Abdolmaleki F, Farahani N, Gheibi Hayat SM, Pirro M, Bianconi V, Barreto GE, et al. The Role of Efferocytosis in Autoimmune Diseases. Front Immunol. 2018;9:1645.

79. Lauber K, Blumenthal SG, Waibel M, Wesselborg S. Clearance of apoptotic cells: getting rid of the corpses. Molecular cell. 2004;14(3):277-87.

80. Serhan CN, Petasis NA. Resolvins and protectins in inflammation resolution. Chemical reviews. 2011;111(10):5922-43. 